\title{
Molecular insights into the humeome of two contrasting soils using HRMS, NMR and GC/TOF MS
}

\author{
Vinci, G. ${ }^{1}$; MAZZei, P. ${ }^{1}$; Piccolo, A. ${ }^{1}$; Bridoux, M. ${ }^{2}$ \\ ${ }^{1}$ Università di Napoli Frederico II, Via Università 100, 80055 \\ Portici, NA, Italy. \\ ${ }^{2}$ CEA, DAM, DIF, 91297 Arpajon, France
}

Soil organic matter (SOM) represents the largest reservoir of organic carbon (OC) in the biosphere, containing approximately $1500 \mathrm{Gt}$ of carbon [1]. This pool of organic matter is mainly composed of humic substances (HS), which consist of a myriad of organic compounds found in soils, whose reactivity and dynamics are directly linked to soil quality and microbial activity, as well as to the soil C sequestration capacity [2]. Recent studies have raised concerns that intensive agricultural practices may significantly contribute to the mineralization of HS, resulting in increased greenhouse gas emissions from soils [3].A better understanding both the composition and dynamics of SOM and its chemically and biologically active fractions are essential for evaluating its vulnerability, in a context of global climate and land-use changes.

The recent acknowledgement of the supramolecular nature of soil HS allowed to devise a fractionation procedure called Humeomics [4] that now enables a comprehensive characterization of the soil humeome at the molecular level. This Humeomics fractionation produces homogeneous fractions by progressively breaking esters and ether C-O bonds but not carbon-carbon bonds. The molecules contained in each fraction are then identified by means of advanced spectroscopic and mass spectrometric techniques. Here, we will compare the humeome of two contrasting French soils, namely (i) a calcaric leptosol and (ii) the podzolic soil of a temperate pine forest, using a combination of high resolution mass spectrometry (ESI and APPI Orbitrap MS), ${ }^{13} \mathrm{C}$ crosspolarization magic-angle-spinning nuclear magnetic resonance ( $\left({ }^{13} \mathrm{C}-\mathrm{CPMAS}-\mathrm{NMR}\right)$ spectroscopy and gaschromatography time-of-flight mass spectrometry (TOF MS) techniques.

[1] Lal (2004) Science 304(5677):1623-1627. [2] Woo et al. (2014) ES\&T 48:12090-12098. [3] Smith et al. (2014) IPCC 816-887. [4] Nebbioso \& Piccolo (2011) Biomacromol 12:1187-1199. 